\title{
Analytical Study of Nonlinear Vibrations of Marine Risers by Newton Harmonic Balance Method
}

\author{
Masoud Rahmani ${ }^{1}$, Amin Moslemi Petrudi ${ }^{1 *}$ \\ ${ }^{1}$ Department of Mechanical Engineering, \\ Tehran University, IRAN \\ *Corresponding Author
}

DOI: https://doi.org/10.30880/jamea.2020.01.02.007

Received 22 May 2020; Accepted 28 June 2020; Available online 31 October 2020

\begin{abstract}
In this paper, the nonlinear motions of marine risers are studied using the Newton's Harmonic Balance Method (NHBM). The nonlinear vibrational equations of the marine risers were obtained in the present study using the Hamilton principle and the Euler-Bernoulli beam theory. The Galerkin's decomposition technique is used to convert the partial differential governing equation (PDE) of the riser vibrations to the ordinary differential equation (ODE). By using the NHBM method, an analytical formulation has been obtained to express the natural nonlinear frequency of the riser. The effect of design parameters such as riser length and initial static displacement of high support has been investigated on riser frequency, which shows acceptable accuracy after comparing the results with previous research. The results show that fluid damping coefficient has a great effect on system instability and reducing this coefficient increases the stability range of the system. Examining the effect of nonlinear parameters shows that the effect of these parameters is greater in large amplitude of motion.
\end{abstract}

Keywords: Newton's Harmonic Balance Method, marine riser, nonlinear vibrations, frequency

\section{Introduction}

Offshore platforms are one of the types of offshore structures used in offshore oil exploration and extraction. The offshore platform can be classified into fixed or floating categories. Marine risers are the most critical and sensitive component of the offshore platform, where the marine risers are used to transfer oil from the seabed to the top of offshore platforms. Figure 1 shows the schematic diagram of a riser pipe with two articulated supports at both ends where the upper joint is subjected to the initial static displacement and the dynamic displacement of the platform [1].

Long structures such as marine risers are known to be nonlinear elastic structures [2, 6]. The critical factor in the design of the marine risers is the estimation of the applied loads on the structure, which have been the subject of many studies [3, 4]. The load exerted from the ocean waves is mainly considered as the main force to be considered in the study of marine structures. Although the force from ocean waves are irregular and unpredictable, they are regularly considered in the design standards [5]. In order to have an accurate estimation of the behavior of such elastic structures, it is necessary to consider the origins of nonlinear term formation in the equation of motion [6]. 

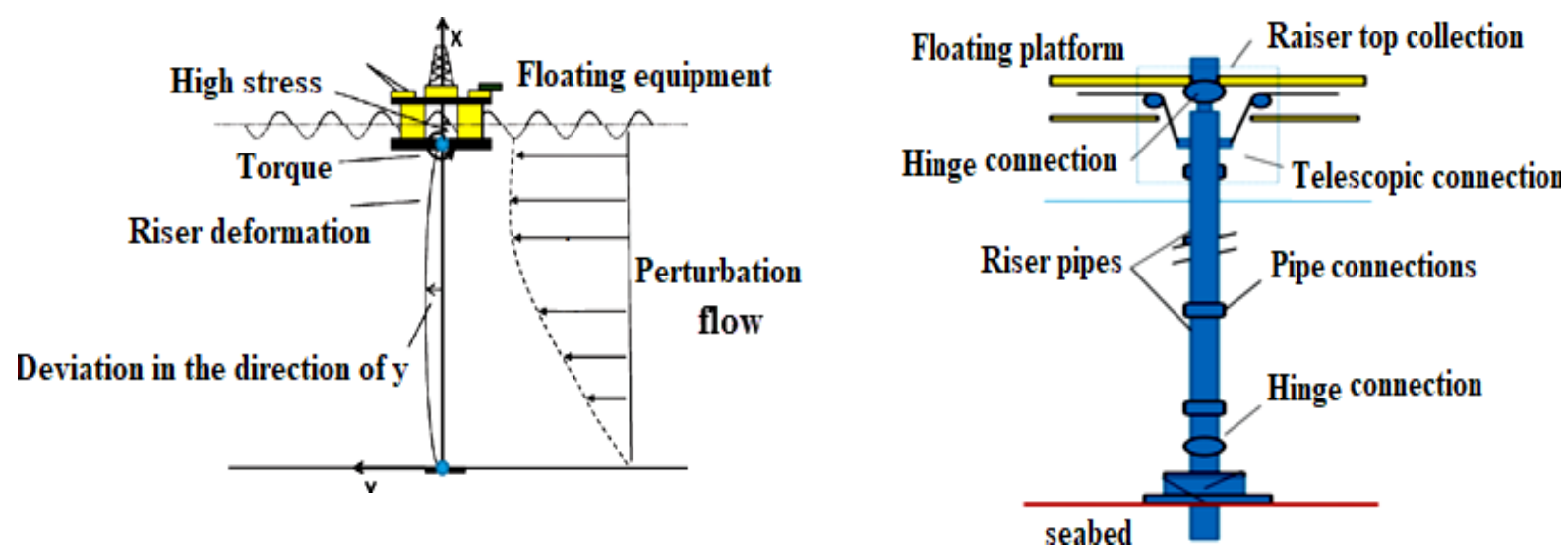

Fig. 1 - Schematic of riser pipe [2]

Nonlinear vibration issues are of great importance in the fields of physics, mechanical structures, and other engineering research. The vibration response, stability, and frequency are vital components of the vibration control system. The examination of the effect of different parameters can therefore be an essential step in the design process. Numerous studies have been conducted in recent years on nonlinear vibrations using analytical and numerical methods to solve complex nonlinear equations. Marcio et al. [7] proposed the use of the error function as an approximation for the nonlinear state of the clearance area to obtain the analytical model according to the LMS algorithm. Chengwu et al. [8] investigate the dynamic analysis of a single degree of freedom mechanical oscillator with preload nonlinearity. In this study, an arctangent function was used to estimate the non-analytical relation of the clearance region. Hakimi et al. [9] investigated the drill string vibration analysis using the differential quadrature method. Barari et al. [10] have solved the problem of nonlinear motion equations using the variational iteration method (VIM) and the Parameter Perturbation Method (PPM). Ansari et al. [11] investigated the geometrically nonlinear free vibration and instability of fluidconveying nanoscale pipes including surface stress effects. Santee et al. [12] studied the oscillations of a beam on a nonlinear elastic foundation under periodic loads. Mazzilli et al. [13] presented the nonlinear equations governing the dynamics of an axial load beam. Khan and Ahmad [14] studied the nonlinear dynamic and bilinear fatigue reliability analysis of marine risers in deep offshore fields. The results related to fatigue reliability and crack size development affecting the fatigue reliability are presented in the work.

In the nonlinear analysis conducted on the riser structure, the bulk of the analysis is based on numerical methods or a combination of numerical and analytical methods. One of the most used methods is the homotopy perturbation method, which involves expanding the series around a small parameter in a nonlinear system. The harmonic balance method is another standard method used in this field to determine the analytical approximations to the periodic solutions of differential equations using a truncated Fourier series. In most cases, the response to natural vibrations of these structures is considered nonlinear and typically represented as the nonlinear equation motion. The nonlinear origin of the structure may be of three types: physical nonlinearity, nonlinear geometry, and nonlinear boundary conditions. In this study, the analysis of nonlinear vibrations of marine risers in stationary waters was performed using the NHBM analysis method. First, a deformation and deflection model of a riser is presented. In this model, the elongation of the middle plane of the riser is considered due to its large amplitude vibrations. The nonlinear effect of fluid damping is also expressed using the Morrison model. The Gallerkin method is used to convert the equivalent of the governing partial differential equation into an ordinary differential equation known as the vibration characteristic equation. In the following section, the analytical relationship has been developed to express the nonlinear frequency of riser vibrations by utilizing a characteristic equivalent of vibrations. The effect of design parameters on riser vibration and stability can be studied by means of the derived analytical formulation.

\section{The Newton Harmonic Balance Method}

NHBM is a combination of the Newton method and the harmonic balance method, which was introduced in 2006 by $\mathrm{Wu}$ et al. [15]. The equation of motion of vibration systems are defined as follows:

$$
\frac{d^{2} x}{d t^{2}}+f\left(u, \frac{d u}{d t}, \frac{d^{2} u}{d t^{2}}\right)=0
$$

where the initial conditions are given as follows:

$$
u(0)=A, \frac{d u}{d t}(0)=0
$$


Assuming that nonlinear function $f$ in the Equation (1) is an odd function, which is: $\left(-u,-\frac{d u}{d t},-\frac{d^{2} u}{d t^{2}}\right)=$ $-f\left(u, \frac{d u}{d t}, \frac{d^{2} u}{d t^{2}}\right)$. By introducing $\omega t=\tau$ in Equations (1), the equation of motion for the vibration system and the initial conditions can be rewritten as follows:

$$
\begin{gathered}
\omega^{2} u^{\prime \prime}+f\left(u, \omega u^{\prime}, \omega^{2} u^{\prime \prime}\right)=0 \\
u(0)=A, u^{\prime}(0)=0
\end{gathered}
$$

In these relations, the $\left(^{\prime}\right)$ symbol shows the differentiation with respect to $\tau$. Using Newton's method, the displacement, $\Delta u_{1}$ and the square of the frequency, $\Delta \omega_{1}^{2}$ are expressed as follows:

$$
\begin{gathered}
u(\tau)=u_{1}(\tau)+\Delta u_{1}(\tau) \\
\omega^{2}=\omega_{1}^{2}+\Delta \omega_{1}^{2}
\end{gathered}
$$

In these relationships, $\Delta u_{1}$ and $\Delta \omega_{1}^{2}$ show a small increase in the principal values of displacement $u_{1}$ and square frequency $\omega_{1}^{2}$, respectively. the analytical approximation of the first order is as follows:

$$
u_{1}(\tau)=A \cos \tau, \quad \Delta u_{1}=\Delta u^{\prime \prime}=\Delta \omega_{1}^{2}=0
$$

For our second approximation, Equation (7) is used after placing Equation (4) and (5) into Equation (3):

$$
\Delta u_{1}=C(\cos \tau-\cos 3 \tau)
$$

By setting the coefficients $\cos 3 \tau$ and $\cos \tau$ to zero, and solving the simultaneous equations in terms of $\mathrm{C}$ and $\Delta \omega_{1}^{2}$, the corresponding second-order approximation for frequency and the approximate periodic displacement solution are obtained as follows $[16,17]$ :

$$
\begin{gathered}
\omega=\sqrt{\omega_{1}^{2}+\Delta \omega_{1}^{2}} \\
u(t)=(A+C) \cos \omega t-C \cos 3 \omega t
\end{gathered}
$$

\section{Extraction and Solution of Motion Equations}

The riser structure is modeled as an elastic beam with length $L$, modulus of elasticity $E$, cross-sectional area $A$ and moment of area $I$ under load $P$ as shown in Fig. 2. The cross-sectional area is assumed to be uniform and the material is homogeneous. The elastic beam is examined according to the Euler-Bernoulli beam theory. Consider an element in the beam with an initial length of $d x$ reaches the length of $d s$ after the displacement and deformation. After the load is applied, point $p$ moves to the $p^{*}$ with the coordinates $\left(X^{*}, Y^{*}\right)$. The displacement is denoted as $u$ in the direction of $x$ and $w$ in the direction of $y$.

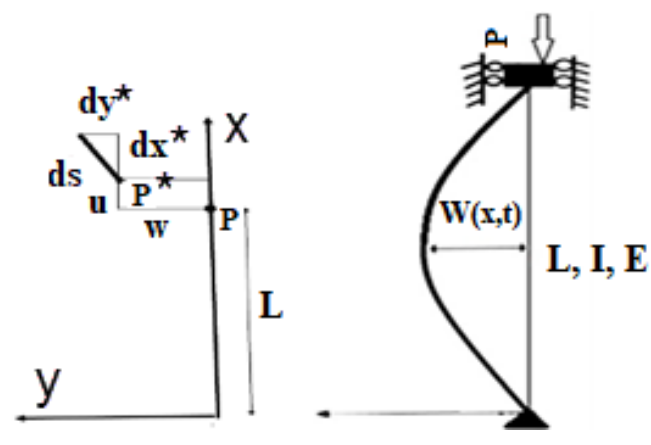

Fig. 2 - Schematic view of a riser and element after deformation and displacement 
Therefore, the parameters defined in Fig. 2 can be expressed by the following equations:

$$
\begin{gathered}
Y^{*}=w, X^{*}=x+u \\
d x^{*}=\left(1+u^{\prime}\right) d x, d y^{*}=w^{\prime} d x \\
d s=\sqrt{d^{2} x^{*}+d^{2} y^{*}}=\sqrt{\left(1+u^{\prime}\right)^{2}+{w^{\prime}}^{2}} d x
\end{gathered}
$$

Therefore, increasing the length of the element, $e$ will be equal to:

$$
\begin{gathered}
e=d s-d x=\sqrt{\left(1+u^{\prime}\right)^{2}+{w^{\prime}}^{2}-1} d x \\
e=\left\{\frac{1}{2}\left[2 u^{\prime}+{u^{\prime}}^{2}+{w^{\prime}}^{2}\right]-\frac{1}{8}\left[2 u^{\prime}+{u^{\prime}}^{2}+w^{\prime 2}\right]+\cdots\right\}
\end{gathered}
$$

Simplify the Equation (14) by ignoring the higher-order terms yields:

$$
e=u^{\prime}+\frac{1}{2} w^{\prime 2}
$$

By integrating Equation (15), the displacement is obtained:

$$
\Delta=u(l)-u(0)+\frac{1}{2} \int_{0}^{l}\left(\frac{\partial w}{\partial x}\right)^{2} d x=\frac{1}{2} \int_{0}^{l}\left(\frac{\partial w}{\partial x}\right)^{2} d x
$$

Hamilton principle is stated as an integral equation in which the energy is integrated over an interval of time. The principle can be stated as the followings:

$$
\delta \int_{t_{1}}^{t_{2}}\left(T-V+W_{N c}\right) d t=0
$$

Next, the parameters required to use the Hamilton principle must be specified.

The expression for the force created in the middle layer is obtained by multiplying the displacement in the hardness of the beam $(\mathrm{AE} / \mathrm{L})$ as follows:

$$
S=\frac{A E}{L} \Delta=\frac{A E}{2 L} \int_{0}^{l}\left(\frac{\partial w}{\partial x}\right)^{2} d x
$$

Kinetic energy is:

$$
T=\frac{1}{2} m \int_{0}^{l}\left(\frac{\partial w}{\partial x}\right)^{2} d x
$$

Bending potential energy:

$$
V_{b}=\frac{1}{2} E I \int_{0}^{l}\left(\frac{\partial^{2} w}{\partial x^{2}}\right)^{2} d x
$$

Energy from the axial force P:

$$
V_{a}=-\frac{1}{4} P \int_{0}^{l}\left(\frac{\partial w}{\partial x}\right)^{2} d x=-\frac{1}{2} P \Delta
$$

Energy from the elongation of the middle layer:

$$
V_{s}=\frac{1}{2} S \Delta=\frac{A E}{8 L}\left(\int_{0}^{l}\left(\frac{\partial w}{\partial x}\right)^{2} d x\right)^{2}
$$

where $T$ is the sum of kinetic energies, $V$ is the sum of potential energies and $W_{N C}$ is nonconservative forces work. By placing Equation (18) - (22) into Equation (17), the following partial differential equation (PDE) is obtained: 


$$
m \frac{\partial^{2} W}{\partial t^{2}}+E I \frac{\partial^{4} W}{\partial x^{4}}+\rho A u^{2} \frac{\partial^{2} W}{\partial x^{2}}+\frac{E A}{L}\left(U_{0}+P(t)+\frac{1}{2} \int_{0}^{L}\left(\frac{\partial V(x . t)}{\partial x}\right)^{2} d x\right) \frac{\partial^{2} W(x . t)}{\partial x}=0
$$

$U_{0}$ indicates the initial static displacement and $P(t)$ shows the dynamic displacement in the upper support.

Next, insert the Gallerkin's method relationship $w(x . t)=\sum_{i=1}^{\infty} \varepsilon \emptyset_{i}(x) z_{i}(t)$ into Equation (23). The function $\emptyset_{i}(x)$ represent the mode shape function of the two-hinge beam. The resulting equation converts the partial derivative motion into an ordinary differential equation, and finally, the equation is obtained in the following form:

$$
\ddot{z}_{(t)}+\left(\omega_{0}^{2}+\delta P(t)\right) z_{(t)}+\beta z_{(t)}^{3}=0
$$

Parameters $\delta, \omega_{0}$ and $\beta$ are defined as follows:

$$
\begin{gathered}
\delta=\frac{E A \pi^{2}}{m L^{2}} \\
\omega_{0}^{2}=\frac{E \pi^{2}\left(I \pi^{2}+A L U_{0}\right)}{m L^{3}}-\frac{\rho A(u \pi)^{2}}{m L^{2}} \\
\beta=\frac{A E \pi^{4}}{4 m L^{4}}
\end{gathered}
$$

The fluid around the riser also applies that force. Assuming that the fluid is stationary, based on the Morrison model for nonlinear fluid damping, the damping force is written as follows [18]:

$$
f_{d}=\varepsilon \dot{Z}(t)|\dot{Z}(t)|
$$

In this equation, $\varepsilon$ is the fluid damping coefficient, which is itself a function of drag coefficient, seawater density, and effective riser level. By adding this assumption to Equation (24), the riser vibration equation will eventually be as follows:

$$
\ddot{z}_{(t)}+\left(\omega_{0}^{2}+\delta P(t)\right) z_{(t)}+\beta z_{(t)}^{3}+\varepsilon \dot{z}(t)|\dot{z}(t)|=0
$$

Equation (27) is the equation governing nonlinear beam vibrations of the Bernoulli Euler, and the center of the beam is subject to the following initial conditions:

$$
z(0)=A, \dot{z}(0)=0
$$

where $A$ denotes the non-dimensional maximum amplitude of oscillation. These conditions indicate that the riser is placed in one of its linear mode shapes with a specific range and then released without applying the initial velocity [19]. Using the harmonic balance method to obtain the frequency equation, and assuming that function $z=A \cos \omega t$ is used in Equation (27), the following equation will be reached:

$$
-A \omega^{2} \cos \omega t+A\left(\omega_{0}^{2}+\delta P(t)\right) \cos \omega t+\beta A^{3}\left(\frac{1}{4} \cos 3 \omega t+\frac{4}{3} \cos \omega t\right)-A \omega \varepsilon \sin \omega t|A \omega \sin \omega t|=0
$$

To get the frequency, $\cos \omega t$ coefficients on both sides of the equation are equal:

$$
\omega=\sqrt{0.75 A^{2} \beta+\omega_{0}^{2}+\delta P(t)}
$$

So the first approximation of the answer to the Equation (24) is $z_{1}=A \cos \left(0.75 A^{2} \beta+\omega_{0}^{2}+\delta P(t)\right)^{1 / 2} t$. In order to obtain the second approximation of Newton's harmonic balance method, placed $\omega t=\tau$ in Equation (24) which yields:

$$
\begin{gathered}
\omega^{2} \ddot{z}_{(t)}+\left(\omega_{0}^{2}+\delta P(t)\right) z_{\tau}+\beta z_{\tau}^{3}=0 \\
z(0)=A \quad z^{\prime}(0)=0
\end{gathered}
$$

By placing the relations (4) and (5) in Equation (30), the following equation can be obtained: 


$$
\left(\omega_{1}^{2}+\Delta \omega_{1}^{2}\right)\left(z_{1}^{\prime \prime}+\Delta z_{1}^{\prime \prime}\right)+\left(\omega_{0}^{2}+\delta P(t)\right)\left(z_{1}+\Delta z_{1}\right)+\beta\left(z_{1}+\Delta z_{1}\right)^{3}=0
$$

Equation (31) can be further linearized with $\Delta u_{1}$ and $\Delta \omega_{1}^{2}$ producing the following form:

$$
z_{1}^{\prime \prime}\left(\omega_{1}^{2}+\Delta \omega_{1}^{2}\right)+\Delta z_{1}^{\prime \prime} \omega_{1}^{2}+\left(\omega_{0}^{2}+\delta P(t)\right)\left(z_{1}+\Delta z_{1}\right)+\beta\left(z_{1}^{3}+3 z_{1}^{2} \Delta z_{1}\right)=0
$$

To get the approximation of the second-order, place the solution of Equation (7) ( $\left.\Delta z_{1}\right)$ in Equation (32) and then obtain the following equations device by setting the coefficients $\cos \tau$ and $\cos 3 \tau$ equal to zero:

$$
\begin{gathered}
-(A+2 C) \omega_{1}^{2}-A \Delta \omega_{1}^{2}+\left(\omega_{0}^{2}+\delta P(t)\right)(A+C)+\frac{4}{3} \beta\left(A^{3}+3 C A^{2}\right)=0 \\
C \omega_{1}^{2}-\left(\omega_{0}^{2}+\delta P(t)\right) C+\frac{1}{4} \beta\left(A^{3}+3 C A^{2}\right)=0
\end{gathered}
$$

Simultaneously solving these equations for both $\Delta \omega^{2}$ and $\mathrm{C}$ leads to the following relations:

$$
\begin{gathered}
C=\frac{-\beta A^{3}}{4 \omega_{1}^{2}-4\left(\omega_{0}^{2}+\delta P(t)\right)+\frac{16}{3} \beta A^{3}} \\
\Delta \omega_{1}^{2}=\frac{\left(\omega_{0}^{2}+\delta P(t)\right)}{A}(A+C)+\frac{4}{3}\left(\beta A^{2}+12 C\right)-\frac{(A+C) \omega_{1}^{2}}{A}
\end{gathered}
$$

Therefore, the approximation of the second order for frequency and displacement by Newton harmonic balance method will be as follows:

$$
\begin{gathered}
Z=(C+A) \cos \omega t-C \cos 3 \omega t=\cos \omega t+\frac{-\beta A^{3}}{4 \omega_{1}^{2}-4\left(\omega_{0}^{2}+\delta P(t)\right)+\frac{16}{3} A}(\cos \omega t-\cos 3 \omega t) \\
\omega=\sqrt{\omega_{1}^{2}+\Delta \omega_{1}^{2}}=\sqrt{\omega_{1}^{2}+\frac{\left(\omega_{0}^{2}+\delta P(t)\right)}{A}(A+C)+\frac{4}{3}\left(\beta A^{2}+12 C\right)-\frac{(A+C) \omega_{1}^{2}}{A}}
\end{gathered}
$$

\section{Results and Discussion}

The results of this method are compared with the homotopy perturbation method [1] and the comparison result presented for different vibration frequencies $(L=20 \mathrm{~m}, 21 \mathrm{~m}$ and $22 \mathrm{~m})$ is shown in Fig. 3. The comparison plot shows that the prediction from the proposed Newton harmonic balance method (NHBM) is consistent with the homotopy perturbation method. Next, the corresponding effect of initial amplitude on the vibration response is given in Fig.4 and Fig.5. The basic parameters used for the simulation of displacement $\mathrm{z}(\mathrm{A}, \mathrm{t})$ are based on values given in Table 1, where the dimensionless coefficient, $\alpha=\pi$ and $\beta=0.15$ are used. Results from Fig. 4 and Fig. 5 demonstrates that the selection of initial amplitude, $A$ would further increase the amplitude of displacement $\mathrm{z}(\mathrm{A}, \mathrm{t})$.

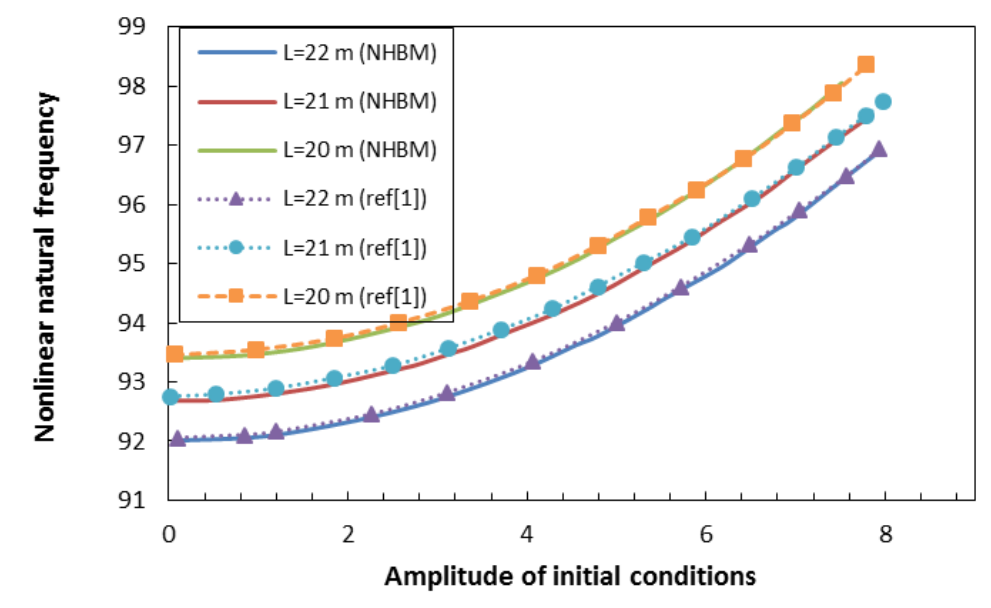

Fig. 3 - Dimensionless current amplitude 
Table 1 - Values of parameters used in the validation

\begin{tabular}{c|c|c}
\hline Parameter & Description & Value \\
\hline L & Riser length & $1000 \mathrm{~m}$ \\
$\mathrm{D}$ & Riser External diameter & $152.4 \mathrm{~mm}$ \\
$\mathrm{~d}$ & Riser inner diameter & $128.4 \mathrm{~mm}$ \\
$\mathrm{EI}$ & Riser stiffness & $1.5 \times 10^{7} \mathrm{Nm}^{2}$ \\
$\varepsilon$ & Fluid damping & $0.5 \mathrm{~N} \mathrm{~s} / \mathrm{m}$ \\
\hline
\end{tabular}

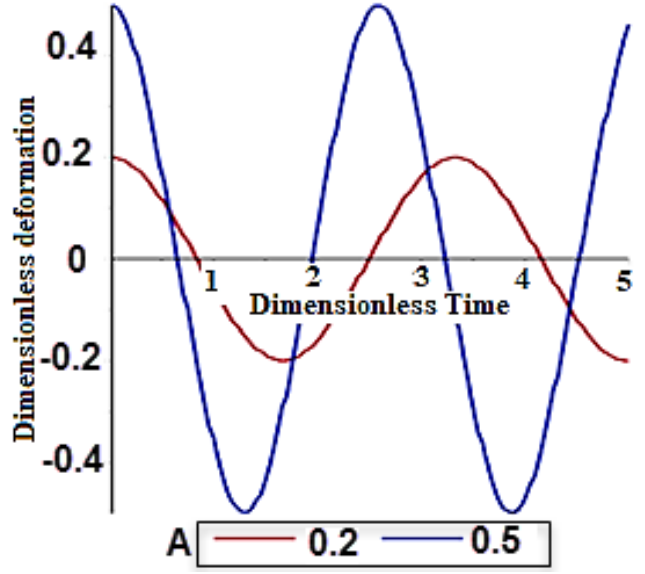

Fig. 4 - The effect of the initial domain on the response

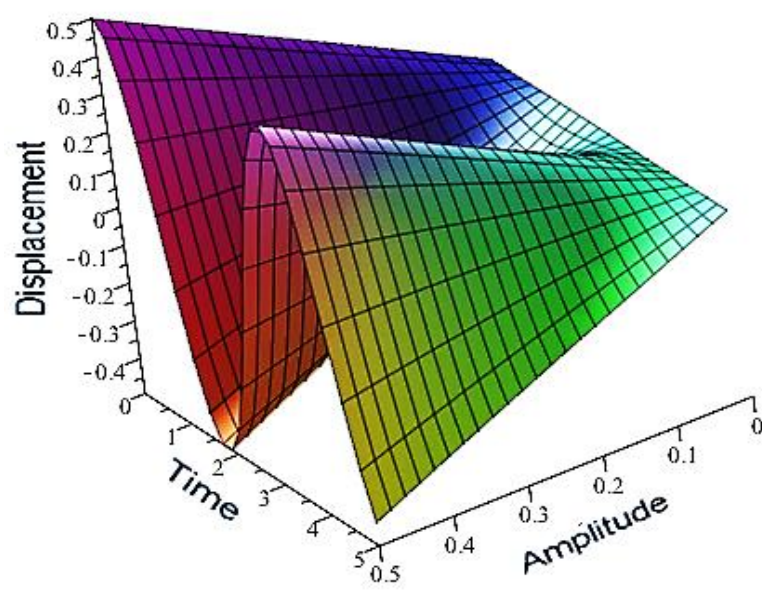

Fig. 5 - The effect of the initial domain on the displacement response relative to time

The Phase Plane diagram and the effect of the parameter $\alpha$ in frequency response are shown in Fig. 6 and Fig. 7. Phase Plane is a graphical and qualitative representation of the characteristics of the differential equation. Important information is obtained from the phase page, including how the system behaves, balance points, stability and instability. In fact, with the help of the phase plate, the qualitative behavior of the differential equation is determined without solving it. Especially in nonlinear systems, which are nonlinear equations, the phase plane drawing is very useful. The phase diagram of this equation under consideration (Equation 27) is shown in Fig. 6, which is quite stable due to the shape of the system without loading (assuming $p(t)=0$ ). The parameter that has the most impact on system stability is the $\varepsilon$ coefficient in damping force. Fig. 8 shows the effect of increasing this coefficient on system instability. $\varepsilon$ is the fluid damping coefficient, which is itself a function of drag coefficient, seawater density, and effective riser level. The effect of the nonlinear factor coefficient $\beta$ on the frequency is shown in Fig. 9.

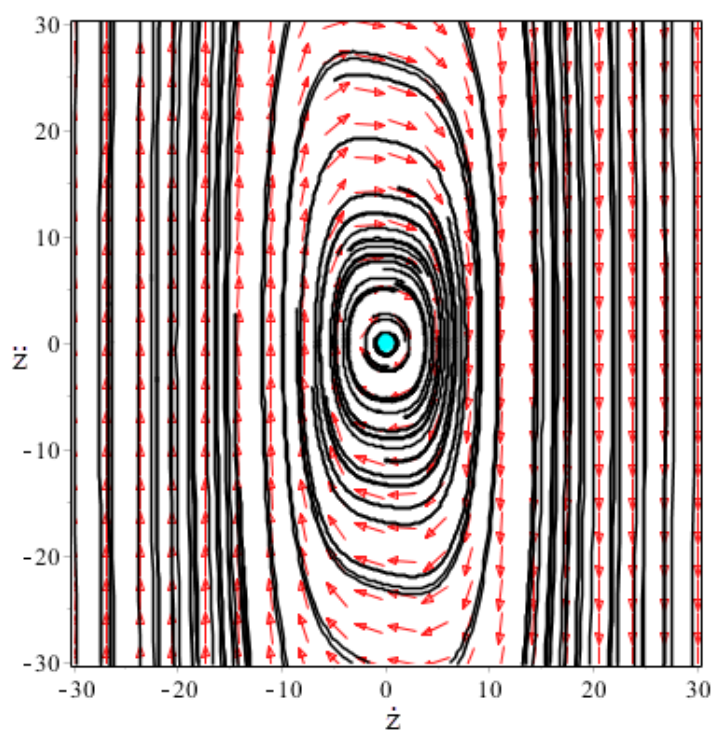

Fig. 6 - Phase Plane $(\beta=0.15, \omega=1, p(t)=0, \varepsilon=0.01)$

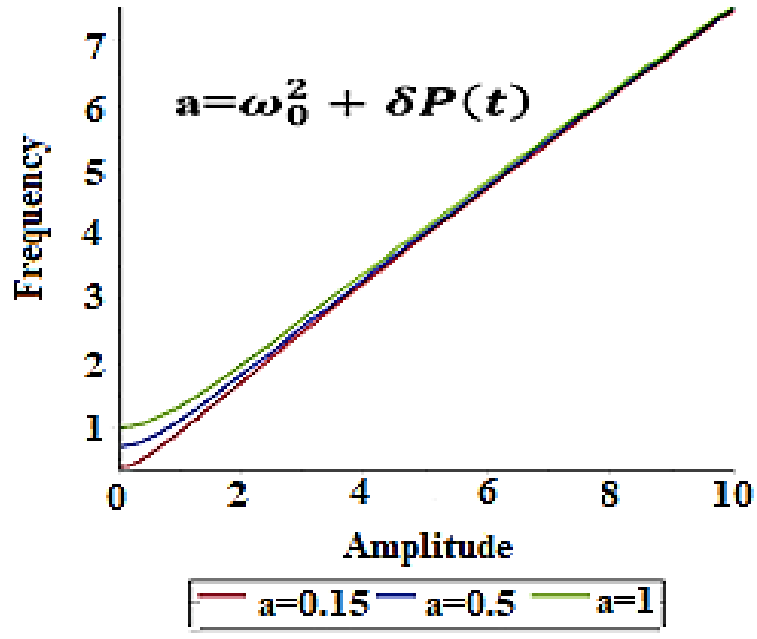

Fig.7 - The effect of changes in $p(t)$ force on vibration frequency 


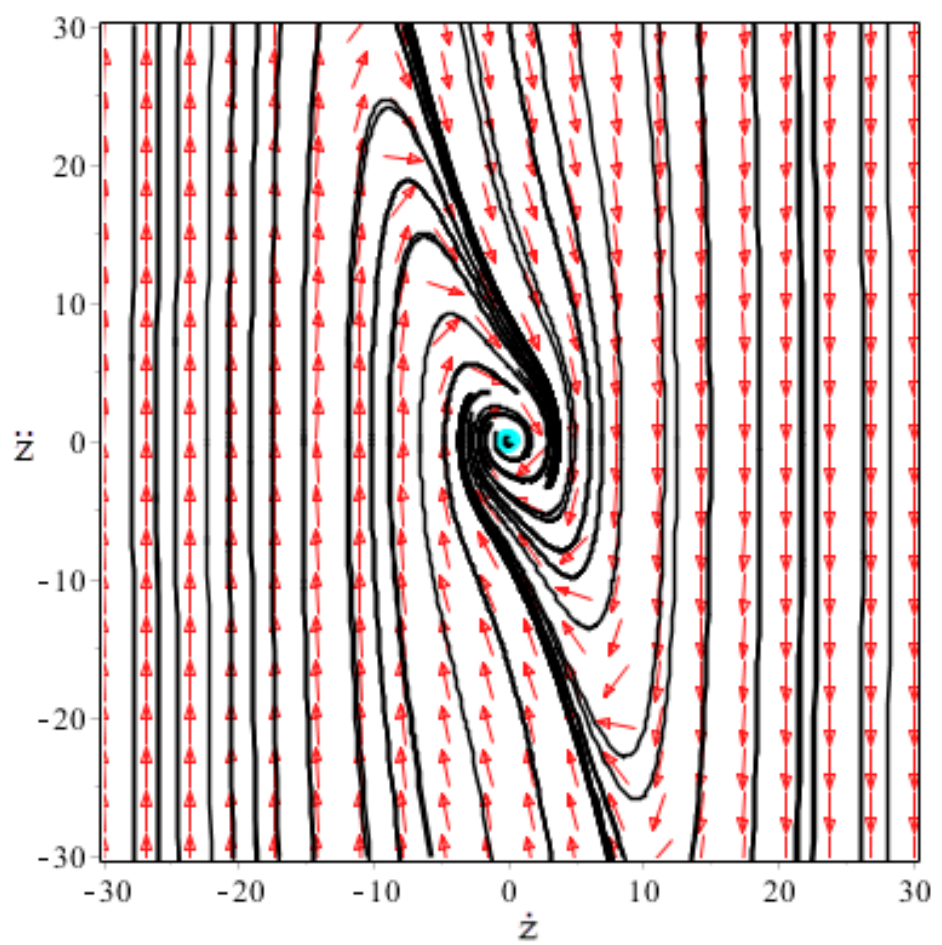

Fig. 8 Phase Plane $(\beta=0.15, \omega=1, p(t)=0, \varepsilon=0.1)$

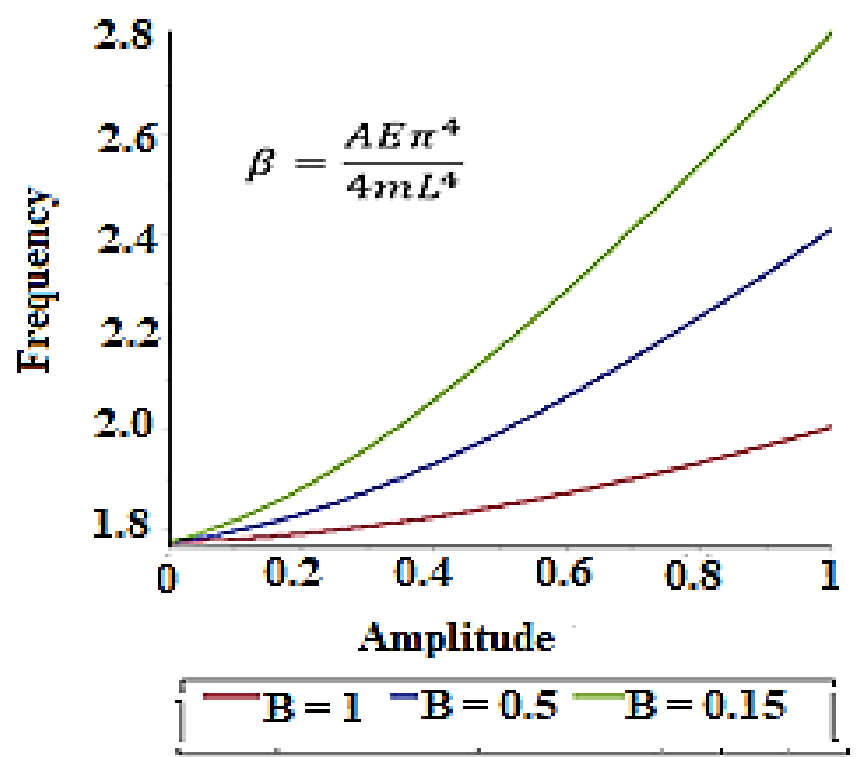

Fig. 9 - Effect of pipe length changes on vibration frequency

\section{Conclusion}

The nonlinear vibration equations of marine risers have been obtained in the present study using the Hamilton principle and the Euler-Bernoulli beam theory. The PDE equation of the vibration problem can be converted into an ordinary differential equation using the Gallerkin method. After obtaining the equation governing the vibrations of the beam, the new Newton Harmonic Balance Method (NHBM) was used to solve this equation. The validation result shows that the proposed method produces similar prediction quality with the homotopy perturbation method. Therefore, it can be concluded that the proposed method could produce satisfactory prediction performance and provide us with the further capability to analyze the vibration problem in terms of various vibration parameters. Results from simulation show that the nonlinear natural frequency of the system decreases as the length of the riser increases. Further findings from the 
simulation also confirm that the fluid damping coefficient has a significant effect on system instability and reducing this coefficient increases the stability range of the system.

\section{Acknowledgement}

The authors would like to thank the Department of Mechanical Engineering, Tehran University, Iran.

\section{References}

[1] Poorjamshidian, Mahmud., Mahjoob Moghadas, Saeid., Mottalebi, Amir Alaeddin., Sheikhi, Javad. "Forced vibration analysis of a nonlinear marine riser using homotopy analysis method." Journal of Marine Engineering 10.19 (2014): 67-74. https:// marine-eng.ir/browse.php?a_id=273\&sid=1\&slc_lang=en

[2] How, B. V. E., S. S. Ge, and Y. S. Choo. "Active control of flexible marine risers." Journal of Sound and Vibration 320, no. 4-5: 758-776, 2009. https://doi.org/10.1016/j.jsv.2008.09.011

[3] Kirk, C. L., E. U. Etok, and M. T. Cooper. "Dynamic and static analysis of a marine riser." Applied Ocean Research 1, no. 3 : 125-135,1979. https://doi.org/10.1016/0141-1187(79)90012-9

[4] Wu, M. C., and J. Y. K. Lou. "Effects of rigidity and internal flow on marine riser dynamics." Applied ocean research 13, no. 5: 235-244, 1991. https://doi.org/10.1016/S0141-1187(05)80047-1

[5] Skjelbreia, Lars, and James Hendrickson. "Fifth order gravity wave theory." Coastal Engineering Proceedings: 10-10, 1960. https://doi.org/10.9753/icce.v7.10

[6] Emam, Samir A. "A theoretical and experimental study of nonlinear dynamics of buckled beams." PhD diss., Virginia Tech, 2002. https://vtechworks.lib.vt.edu/handle/10919/25970

[7] Costa, Márcio Holsbach, Leandro Ronchini Ximenes, and José Carlos Moreira Bermudez. "Statistical analysis of the LMS adaptive algorithm subjected to a symmetric dead-zone nonlinearity at the adaptive filter output." Signal Processing 88, no. 6: 1485-1495, 2008. https://doi.org/10.1016/j.sigpro.2007.12.008

[8] Duan, Chengwu, and Rajendra Singh. "Dynamic analysis of preload nonlinearity in a mechanical oscillator." Journal of Sound and Vibration 301, no. 3-5 : 963-978, 2007. https://doi.org/10.1016/j.jsv.2006.10.042

[9] Hakimi, H., and S. Moradi. "Drillstring vibration analysis using differential quadrature method." Journal of Petroleum Science and Engineering 70, no. 3-4 : 235-242, 2010. https://doi.org/10.1016/j.petrol.2009.11.016

[10] Barari, Amin, Hamed Dadashpour Kaliji, Mojtaba Ghadimi, and G. Domairry. "Nonlinear vibration of EulerBernoulli beams." Latin American Journal of Solids and Structures 8, no. 2: 139-148, 2011. https://doi.org/10.1590/S1679-78252011000200002

[11] Ansari, R., A. Norouzzadeh, R. Gholami, M. Faghih Shojaei, and M. A. Darabi. "Geometrically nonlinear free vibration and instability of fluid-conveying nanoscale pipes including surface stress effects." Microfluidics and Nanofluidics 20, no. 1: 28, 2016. https://link.springer.com/article/10.1007/s10404-015-1669-y

[12] Santee, Donald Mark, and Paulo Batista Gonçalves. "Oscillations of a beam on a nonlinear elastic foundation under periodic loads." Shock and Vibration 13, no. 4-5: 273-284, 2006. https://doi.org/10.1155/2006/534593

[13] Mazzilli, Carlos EN, César T. Sanches, Odulpho GP Baracho Neto, Marian Wiercigroch, and Marko Keber. "Nonlinear modal analysis for beams subjected to axial loads: Analytical and finite-element solutions." International Journal of Non-Linear Mechanics 43, no. 6: 551-561, 2008. https://doi.org/10.1016/j.ijnonlinmec.2008.04.004

[14] Khan, Rizwan A., and Suhail Ahmad. "Nonlinear dynamic and bilinear fatigue reliability analyses of marine risers in deep offshore fields." Ships and Offshore Structures 13, no. 1: 10-19, 2018. https://doi.org/10.1080/17445302.2017.1328755

[15] Wu, B. S., W. P. Sun, and C. W. Lim. "An analytical approximate technique for a class of strongly nonlinear oscillators." International Journal of Non-Linear Mechanics 41, no. 6-7: 766-774, 2006. https://doi.org/10.1016/j.ijnonlinmec.2006.01.006

[16] Mashinchi Joubari, Mohammad Mehdi, Mohammad Hadi Pashaei, and Hamid Javaniyan Jouybari. "Solution of strongly nonlinear oscillator problem arising in Plasma Physics with Newton Harmonic Balance Method." Journal of Applied and Computational Mechanics 1, no. 2: 59-66, 2014. http://jacm.scu.ac.ir/article_10756_1.html

[17] Santee, Donald Mark, and Paulo Batista Gonçalves. "Oscillations of a beam on a non-linear elastic foundation under periodic loads." Shock and Vibration 13, no. 4-5: 273-284, 2006. https://doi.org/10.1155/2006/534593

[18] Mazzilli, Carlos EN, César T. Sanches, Odulpho GP Baracho Neto, Marian Wiercigroch, and Marko Keber. "Non-linear modal analysis for beams subjected to axial loads: Analytical and finite-element solutions." International Journal of Non-Linear Mechanics 43, no. 6: 551-561, 2008. https://doi.org/10.1016/j.ijnonlinmec.2008.04.004

[19] Wu, B. S., W. P. Sun, and C. W. Lim. "An analytical approximate technique for a class of strongly non-linear oscillators." International Journal of Non-Linear Mechanics 41, no. 6-7: 766-774, 2006 
https://doi.org/10.1016/j.ijnonlinmec.2006.01.006

[20] Mashinchi Joubari, Mohammad Mehdi, Mohammad Hadi Pashaei, and Hamid Javaniyan Jouybari. "Solution of strongly nonlinear oscillator problem arising in Plasma Physics with Newton Harmonic Balance Method." Journal of Applied and Computational Mechanics 1, no. 2: 59-66, 2014.

http://jacm.scu.ac.ir/article_10756_1.html

[21] Rubino, A., Matteo Pini, P. Colonna, T. Albring, S. Nimmagadda, T. Economon, and J. Alonso. "Adjoint-based fluid dynamic design optimization in quasi-periodic unsteady flow problems using a harmonic balance method." Journal of Computational Physics 372: 220-235, 2018. https://doi.org/10.1016/j.jcp.2018.06.023

[22] Kaewunruen, Sakdirat, Julapot Chiravatchradej, and Somchai Chucheepsakul. "Nonlinear free vibrations of marine risers/pipes transporting fluid." Ocean Engineering 32, no. 3-4 : 417-440, 2005. https://doi.org/10.1016/j.oceaneng.2004.07.007

[23] Malatkar, Pramod. "Nonlinear vibrations of cantilever beams and plates." PhD diss., Virginia Tech, 2003. https://vtechworks.lib.vt.edu/handle/10919/28301 